\title{
OBESIDAD ABDOMINAL Y AUSENTISMO POR CAUSA MÉDICA EN UNA EMPRESA DE LA INDUSTRIA METALMECÁNICA EN CALI, COLOMBIA
}

\author{
Ricardo A. Agredo Zúñiga1,2,a, Emily S. García Ordoñez,b, Carlos Osorioº,c, Natalia Escudero ${ }^{4, b}$, \\ Carlos A. López-Albán ${ }^{4, e}$, Robinson Ramírez-Vélez ${ }^{5, f}$
}

\section{RESUMEN}

Con el objetivo de examinar la frecuencia de obesidad abdominal y el ausentismo laboral por causa médica, se realizó un estudio transversal en 185 varones pertenecientes a una industria metalmecánica. En ellos, además de datos sociodemográficos, se recogió datos antropométricos y se midió la circunferencia de cintura (CC) en centímetros como indicador de obesidad abdominal. Se encontró, además de una alta frecuencia de obesidad abdominal $(28,7 \%)$, que los trabajadores con obesidad abdominal presentaron mayor frecuencia, mayor tiempo y mayores costos por las incapacidades médicas. Se concluye que la obesidad abdominal en esta muestra fue altamente frecuente; no obstante, al tratarse de un riesgo reversible, las empresas podrían beneficiarse, en términos de ausentismo laboral, de programas preventivo promocionales destinados a controlar este problema.

Palabras clave: Obesidad abdominal; Ausentismo; Salud laboral (fuente: DeCS - BIREME).

\section{ABDOMINAL OBESITY ASSOCIATED TO MEDICAL-RELATED ABSENTEEISM AT A COMPANY OF METAL-MECHANICAL INDUSTRY IN CALI, COLOMBIA}

\section{ABSTRACT}

The aim of this study was examined the abdominal obesity prevalence and association with medical-related absenteeism. A cross-sectional study in 185 men from the metal-mechanical industry was conducted. Sociodemografic and antropometrics data of was gathered, waist circumference was measured as an indicator of abdominal obesity. The prevalence of central obesity was $28.7 \%$. Subjects with higher values of central obesity showed higher frequency, higher duration and higher costs of medical-related absenteeism, however, this relationship was not significant. We conclude that abdominal obesity is highly frequent in this sample; however, since it's a reversible risk, enterprises could get benefits from preventive and promotional programs destiny to control this problem.

Key words: Obesity, abdominal; Absenteeism; Occupational health (source: MeSH - NLM).

\section{INTRODUCCIÓN}

La obesidad ha sido reconocida como un problema de salud pública en todo el mundo (1). El aumento de su prevalencia ha alcanzado caracteres epidémicos en la gran mayoría de los países industrializados y en desarrollo ${ }^{(1,2)}$. En 2008, el estudio INTERHEART señaló que en países de Latinoamérica y el Caribe, uno de cada dos adultos tenía exceso de peso (sobrepeso u obesidad), el cual se expresaba más comúnmente por un incremento de la circunferencia de cintura (CC ${ }^{(2)}$. De igual manera dos estudios epidemiológicos, ejecutados en más de 20 países de medianos y bajos ingresos, incluyendo Colombia, han señalado que la obesidad abdominal es el factor más

\footnotetext{
Grupo de Investigación Movimiento Humano y Salud (GIMHUS), Programa de Fisioterapia, Facultad de Ciencias de la Salud, Universidad de San Buenaventura. Cartagena, Colombia.

2 Fisioterapia Integral para Todos FIT. Cali, Colombia.

3 Escuela de Salud Pública, Universidad del Valle. Cali, Colombia.

4 Centro para la Investigación en Salud y Rendimiento Humano ZOE. Cali, Colombia.

Grupo de Investigación en Ciencias Aplicadas al Ejercicio Físico, el Deporte y la Salud (GICAEDS), Facultad de Cultura Física, Deporte y Recreación, Universidad Santo Tomás. Bogotá, Colombia.

a Fisioterapeuta máster en Salud Ocupacional; ${ }^{\mathrm{b}}$ fisioterapeuta; ${ }^{\mathrm{c}}$ médico máster en Salud Ocupacional; ${ }^{\mathrm{e}}$ médico máster en Salud Pública; ${ }^{\mathrm{f}}$ fisioterapeuta PhD en Ciencias Biomédicas.

Recibido: 13-12-12 Aprobado: 08-05-13
}

Citar como: Agredo Zúñiga RA, García Ordoñez ES, Osorio C, Escudero N, López-Albán CA, Ramírez-Vélez R. Obesidad abdominal y ausentismo por causa médica en una empresa de la industria metalmecánica en Cali, Colombia. Rev Peru Med Exp Salud Publica. 2013;30(2):251-5. 
preponderante en los países Latinoamericanos y el que mejor explica la ocurrencia de un primer infarto agudo de miocardio (IAM) o de un primer accidente cerebro-vascular $(\mathrm{ACV})$ isquémico o hemorrágico ${ }^{(2,3)}$.

Por otro lado, se ha señalado que la obesidad aumenta de manera significativa los costos en salud y el ausentismo laboral en población hispana por todas las causas ${ }^{(4)}$. Asimismo, se ha descrito que la obesidad se encuentra relacionada con una alta demanda psicológica ${ }^{(5)}$ y con un incremento en el número de trastornos musculo esqueléticos ${ }^{(6)}$. En Colombia, las ausencias por enfermedad, o como se define con mayor precisión en el Sistema General de Riesgos Laborales de este país: la ausencia al trabajo que se le atribuye a la enfermedad que no se catalogada como laboral (7), ocupa las tres cuartas partes del ausentismo total en la industria general y es un problema no resuelto por los gobiernos y las empresas en todo el mundo ${ }^{(6-8)}$.

En la literatura biomédica existen estudios que han demostrado que el incremento de la CC se asocia con mayor morbilidad o mortalidad por múltiples causas ${ }^{(3,4,9,10)}$, pero solo unos pocos estudios han estudiado la relación entre la obesidad abdominal y la ausencias por enfermedad ${ }^{(8-12)}$. Por tanto, el objetivo de este estudio fue examinar la prevalencia de obesidad abdominal y su asociación con el ausentismo laboral por causa médica.

\section{EL ESTUDIO}

Se desarrolló un estudio transversal en trabajadores de una industria metalmecánica de Cali (Colombia). Se incluyeron a todos los trabajadores entre 18 y 65 años que hayan referido un historial libre de enfermedades cardiovasculares o endocrinas, y que presentaran el informe completo de ausentismo laboral del año 2011. Se excluyeron a los trabajadores que, según el sistema de gestión de la seguridad y salud en el trabajo, presentaran: enfermedad crónica o inflamatoria: diagnóstico previo de diabetes mellitus tipo 1 o 2: procesos infecciosos: cualquier enfermedad que afecte el metabolismo de la glucosa o los lípidos: antecedentes de cualquier procedimiento quirúrgico o trauma mayor a 12 meses antes del estudio; alteración cardiaca significativa, y sujetos con alteraciones neurológicas, psiquiátricas o limitación cognitiva que no les permitiera consentir su participación en el estudio. Se incluyó a todos los trabajadores, por lo que no se realizó ninguna estrategia de muestreo.

\section{RECOLECCION DE LOS DATOS}

A cada individuo se le realizó un examen médico y una encuesta de salud, donde se registraron datos sociodemográficos, antecedentes personales y familiares patológicos, una encuesta de predicción de capacidad física en unidades metabólicas (MET) ${ }^{(12)}$ y una encuesta de estilos de vida, validada en población Colombiana (13). Posteriormente, de cada participante se registró la talla en posición erguida con un antropómetro Krammer ${ }^{\circledR}$ (Holtain Ltd., Crymych Dyfed, RU) de cuatro segmentos y $1 \mathrm{~mm}$ de precisión. El peso se midió con balanzas de piso Health-o-Meter ${ }^{\circledR}$ (Continental Scale Corp., Bridgeview, EE. UU.) con $500 \mathrm{~g}$ de precisión, calibradas con pesos conocidos. El índice de masa corporal (IMC) se obtuvo dividiendo el peso entre el cuadrado de la talla ${ }^{(14)}$.

Para conocer la frecuencia de obesidad abdominal se midió la $\mathrm{CC}$ en centímetros en un punto intermedio entre la última costilla y la cresta ilíaca, con el sujeto de pie al final de una espiración suave y se clasificó la población según los criterios señalados por la Federación Internacional de Diabetes (IDF) ${ }^{(15)}$, los que señalan que una CC mayor de $90 \mathrm{~cm}$ en varones es considerada como criterio de obesidad abdominal.

La Gerencia de Recursos Humanos y Salud Ocupacional de la empresa permitió el acceso a la información y registros del ausentismo laboral de los trabajadores evaluados en el periodo de estudio, donde se detalla con precisión las causas (accidente de trabajo, enfermedad profesional, enfermedad general etc.), la frecuencia (número de personas), la duración promedio (en horas) y los costos (en dólares americanos por hora) de cada incapacidad presentada en los trabajadores en el periodo de estudio. Se incluyeron en el análisis de costos únicamente los gastos económicos directos para la empresa, derivado de las incapacidades por causa médica (correspondiente al 100\% del valor de la incapacidad durante los tres primeros días, y superado este periodo, un $33 \%$ del valor de la incapacidad) ${ }^{(7)}$.

\section{PROCESAMIENTO Y ANÁLISIS DE DATOS}

Mediante la prueba de Kolmogorov-Smirnov se rechazó la hipótesis de distribución normal para las variables estudiadas. El análisis univariado incluyó medidas de tendencia central y dispersión. Se incluyeron únicamente las incapacidades por causas médicas derivadas de enfermedades osteomusculares y respiratorias, según la Clasificación Internacional de Enfermedades (CIE10), por ser las de mayor frecuencia en la empresa. Se utilizó la prueba de chi cuadrado y la prueba $U$ de MannWhitney para comparar la frecuencia, la duración y los costos de las incapacidades por causa médica, según la CC y la distribución de las variables, respectivamente. Los resultados se analizaron en el paquete estadístico Statistic Package for the Social Sciences (SPSS) versión 
20 para Windows, y un valor $p<0,05$ se consideró como significante.

\section{ASPECTOS ÉTICOS}

El Comité de Ética de Investigación en Seres Humanos de la Universidad del Valle, veló por el cumplimiento de los aspectos éticos y de protección de la privacidad de los sujetos. Se hizo un muestreo por conveniencia teniendo en cuenta que la participación de los trabajadores fue voluntaria previa firma de consentimiento informado.

\section{HALLAZGOS}

La edad promedio de los participantes fue de 33,9 $\pm 9,3$ años. A pesar que la media de las variables antropométricas y clínicas estudiadas sugiere que la mayor parte de la población se encuentra aparentemente sana; un 38,4 \% de los trabajadores refirió, al menos, una ausencia por causa médica (media 17,9 $\pm 53,2 \mathrm{~h}$ ) en el periodo estudiado (Tabla 1), el detalle de otras

Tabla 1. Distribución de las variables demográficas y del estado de salud de la población evaluada, $(n=185)$

\begin{tabular}{|c|c|}
\hline Variables & $\mathrm{N} .{ }^{\circ}(\%)$ \\
\hline \multicolumn{2}{|l|}{ Variables antropométricas } \\
\hline Edad (años) & $33,9(9,3)^{*}$ \\
\hline Peso (kg) & $70,3(10,3)^{*}$ \\
\hline Talla (cm) & $169,4(7,1)^{*}$ \\
\hline IMC $\left(\mathrm{kg} / \mathrm{m}^{2}\right)$ & $24,4(3,1)^{*}$ \\
\hline $\mathrm{CC}(\mathrm{cm})$ & $81,5(9,0)^{*}$ \\
\hline Capacidad física (Mets) & $11,8(1,6)^{*}$ \\
\hline Estilo de vida (test fantástico) & $81,6(9,3)^{*}$ \\
\hline Horas de incapacidad & $17,9(53,2)^{*}$ \\
\hline \multicolumn{2}{|l|}{ Raza } \\
\hline Afrodescendiente & $46(24,9)$ \\
\hline Indígena & $14(7,6)$ \\
\hline Mestizo & $74(40,0)$ \\
\hline Blanco & $48(25,9)$ \\
\hline Otros & $3(1,6)$ \\
\hline \multicolumn{2}{|l|}{ Estado civil } \\
\hline Soltero & $60(32,4)$ \\
\hline Viudo & $2(1,1)$ \\
\hline Separado & $20(10,8)$ \\
\hline Casado & $47(25,4)$ \\
\hline Unión libre & $55(29,7)$ \\
\hline No sabe / No responde & $1(0,5)$ \\
\hline \multicolumn{2}{|l|}{ Nivel educativo } \\
\hline Ninguno & $3(1,6)$ \\
\hline Primaria & $4(22)$ \\
\hline Bachillerato & $101(54,6)$ \\
\hline Técnico & $55(29,7)$ \\
\hline Universitario & $22(11,9)$ \\
\hline
\end{tabular}

* Media \pm desviación estándar

CC: circunferencia de cintura variables sociodemográficas se muestra en la Tabla 1. Un $28,7 \%$ de los trabajadores presentó obesidad abdominal. Se observó una mayor frecuencia de incapacidad laboral entre los trabajadores con obesidad abdominal en comparación con los no obesos (42 y $36 \%$ respectivamente $[p=0,78$, chi cuadrado]); de igual forma, mayor tiempo de incapacidad laboral (21,7 \pm 9,2 y $16,3 \pm 10,5$ horas respectivamente $[p=0,59, U$ de Mann-Whitney]) y mayor costo por hora de incapacidad médica $(22,9 \pm 11,8$ y 17,4 $\pm 7,7$ dólares americanos por hora $[p=0,63, U$ de Mann-Whitney]). Las principales causas de incapacidad médica fueron las enfermedades asociadas al sistema osteomuscular y respiratorio, con un total de 3312 horas de incapacidad en el año 2011, las que equivalen al 53,9 y $59,7 \%$ de la incapacidad presentada en la empresa por todas las causas y por enfermedad general, respectivamente.

\section{DISCUSIÓN}

La evidencia epidemiológica y experimental demuestra que el sobrepeso y la obesidad son una epidemia mundial con tendencia ascendente ${ }^{(1-3)}$. En América Latina también se está produciendo un rápido y alarmante incremento en la prevalencia de obesidad, debido principalmente a factores como el crecimiento económico; la urbanización progresiva, y el consiguiente cambio en los estilos de vida, que involucra un mayor consumo de alimentos de alta densidad energética, el abandono de dietas tradicionales, y la disminución generalizada de los niveles de actividad física ${ }^{(1,3,11)}$. Según la Encuesta Nacional de la situación Nutricional en Colombia 2010 (ENSIN 2010) ${ }^{(16)}$, el 39\% de hombres entre 18 y 64 años presentó obesidad abdominal, lo cual supera en un $11 \%$ la frecuencia encontrada en este trabajo, no obstante, estos hallazgos son alarmantes dado el incremento en la morbilidad y la mortalidad prematura asociados con obesidad (17-19). Muchos estudios han señalado que el incremento de la CC se encuentra asociado con morbilidad y mortalidad por todas las causas, especialmente la enfermedad cardiovascular y metabólica; por lo cual consideramos que el problema de la obesidad merece una especial atención no solo por parte de las autoridades de salud en la empresa en la cual se llevó a cabo este estudio, sino que además las autoridades vinculadas a salud ocupacional y salud publica debieran participar del análisis, control, evaluación y supervisión de este problema.

Existen pocos estudios, como los de Grunstein et al. ${ }^{(5)}$ y Larsson et al. (9), que han encontrado una asociación significativa entre la obesidad abdominal y las ausencias laborales por enfermedad. Si bien no se 
encontró significancia estadística en los indicadores de ausentismo laboral considerados en este estudio, se observa mayor frecuencia por incapacidad $(7 \%)$, mayor duración promedio (5,4 horas) y un incremento en los costos médicos (5,5 dólares americanos) en los trabajadores con mayor CC. Este hallazgo coincide con lo reportado por Moreau et al. (20), en un grupo de 20 463 trabajadores pertenecientes a 25 compañías en Bélgica, quienes encontraron que varones con mayores valores en la CC, mostraban un incremento en los periodos de ausencia laboral y mayores costos en las incapacidades por todas las causas de enfermedad general, en comparación con los empleados con una CC considerada como saludable.

Aun cuando en este estudio no se pudo evaluar la asociación entre la obesidad abdominal y el ausentismo por causa médica, esta sí fue descrita en el estudio de Tucker y Friedman ${ }^{(8)}$, quienes estudiaron el impacto de la obesidad sobre la productividad laboral, en más de 10000 empleados de 50 empresas, encontrando que los individuos obesos tenían 1,7 veces la probabilidad de experimentar alto ausentismo (siete o más ausencias por enfermedad en los últimos seis meses), y tenían 1,6 veces mayor riesgo de experimentar ausentismo moderado (tres a seis ausencias en los últimos seis meses) en comparación con sus pares no obesos $(p=0,05)$.

Algunos aspectos deben ser tomados en cuenta como limitantes del estudio. Por ejemplo, el tamaño de la muestra, las características propias de la población, el diseño del trabajo y el tipo de muestreo, pueden introducir sesgos; tampoco se incluyeron otras variables que pueden estar asociados al ausentismo laboral, como son los riesgos psicosociales. Se ha informado que la depresión, la ansiedad y el estrés, se relacionan con alteraciones funcionales, pérdida de la capacidad de trabajo y un incremento del ausentismo laboral (8). Sin embargo, se observó congruencia de los resultados con datos reportados en otros estudios nacionales e internacionales $(10,13,16,19,20)$.

En conclusión se encontró, una alta frecuencia de obesidad abdominal en la población de estudio, observándose mayor frecuencia de incapacidad laboral, mayor tiempo de incapacidad laboral y mayor costo por hora de incapacidad médica entre los trabajadores con obesidad abdominal, en comparación con los no obesos, aun cuando estas diferencias no fueron significativas. No obstante, al ser la obesidad un riesgo reversible, los empresarios podrían beneficiarse de programas destinados a la prevención y el tratamiento de la obesidad, incluyendo la promoción de estilos de vida saludables como estrategia terapéutica, por su impacto significativo en la salud pública, en la productividad laboral y en el bienestar de los trabajadores.

Contribuciones de autoría: RAA y RRV participaron en la concepción y diseño del artículo; RAA, ESG y NE en la recolección y obtención de resultados, análisis e interpretación de datos. RAA, CAL y CO brindaron asesoría estadística. ESG, CAL y CO brindaron asesoría técnica o administrativa y han participado en el trabajo de campo. RAA y RRV participaron en la redacción del artículo. Todos los autores participaron en la revisión crítica del artículo y aprobación de su versión final.

Fuentes de financiamiento: autofinanciado.

Conflictos de interés: los autores declaran no tener ningún conflicto de interés.

\section{REFERENCIAS BIBLIOGRÁFICAS}

1. National Institutes of Health. Clinical guidelines on the identification, evaluation, and treatment of overweight and obesity in adults. Executive summary. Bethesda, Md: National Institutes of Health, National Heart, Lung, and Blood Institute; 1998.

2. Lanas F. Factores de riesgo cardiovascular en América Latina: estudio INTERHEAR. Medwave. 2008;8(10):e1785. doi: 10.5867/ medwave.2008.10.1785.

3. O’Donnell MJ, Xavier D, Liu L, Zhang H, Chin SL, Rao-Melacini P, et al. Risk factors for ischaemic and intracerebral haemorrhagic stroke in 22 countries (the INTERSTROKE study): a case-control study. Lancet. 2010;376(9735):112-23.

4. Zarate A, Crestto M, Maiz A, Ravest G, Pino MI, Valdivia G, et al. Influencia de la obesidad en los costos en salud y en el ausentismo laboral de causa médica en una cohorte de trabajadores. Rev Méd Chile. 2009;137(3):337-44.

5. Hellerstedt WL, Jeffery RW. The association of job strain and health behaviours in men and women. Int $\mathrm{J}$ Epidemiol. 1997;26(3):575-83.

6. Ricci JA, Chee E. Lost productive time associated with excess weight in the U.S. workforce. J Occup Environ Med. 2005;47(12):1227-34.
7. Colombia, Ministerio de Trabajo. Ley 1562 del 11 de Julio de 2012 "Por la cual se modifica el sistema de riesgos laborales y se dictan otras disposiciones en materia de salud ocupacional".

8. Tucker LA, Friedman GM. Obesity and absenteeism: an epidemiologic study of 10,825 employed adults. Am J Health Promot. 1998;12(3):202-7.

9. Larsson B. Regional obesity as a health hazard in men--prospective studies. Acta Med Scand Suppl 1988;723:45-51.

10. Suárez-Ortegón MF, Arbeláez A, Mosquera M, Aguilar de Plata AC, Ramírez-Vélez R. Los niveles de ferritina y los marcadores de riesgo 
cardiovascular se correlacionan con mayor tiempo sedentario autoreportado en hombres aparentemente sanos. Rev Colomb Cardiol. 2012;19(1):4-10.

11. Visscher TL, Seidell JC. The public health impact of obesity. Annu Rev Public Health. 2001;22:355-75.

12. Ramírez-Vélez R, Delgado P. Análisis comparativo de las ecuaciones desarrolladas por Jackson et al y por el American College of Sports Medicine (ACSM) para predecir el consumo máximo de oxígeno en estudiantes de fisioterapia. Fisioterapia. 2008;30(1):24-33.

13. Ramírez-Vélez R, Agredo RA. Fiabilidad y validez del instrumento "Fantástico" para medir el estilo de vida en adultos colombianos. Rev Salud Pública. 2012;14(2):226-37.

14. López-Albán CA, Ramírez-Vélez R, Sánchez-Gallardo CE, Marmolejo LC. Características antropométricas y funcionales de individuos físicamente activos. Iatreia. 2008;21(2):121-8.
15. Saely CH, Rein P, Drexel H. The metabolic syndrome and risk of cardiovascular disease and diabetes: experiences with the new diagnostic criteria from the International Diabetes Federation. Horm Metab Res. 2007;39(9):642-50.

16. Colombia, Ministerio de la Protección Social, Instituto Nacional de Salud, Instituto Colombiano de Bienestar Familiar. Encuesta Nacional de la Situación Nutricional en Colombia (ENSIN) 2010. Libro Digital. Bogota: Ministerio de la Protección Social; 2010.

17. Dosman VA, Triviño LP, Uribe YL, Agredo RA, Jerez AM, Ramírez R. Asociación entre la aptitud física y los factores de riesgo de síndrome metabólico en trabajadores de una institución universitaria. Rev Colomb Cardiol. 2009; 16(4):153-8.

18. Ramírez R, Agredo RA. El sedentarismo es un factor predictor de hipertrigliceridemia, obesidad central y sobrepeso. Rev Colomb Cardiol. 2012;19(2):75-9.
19. Flegal KM, Graubard BI. Estimates of excess deaths associated with body mass index and other anthropometric variables. Am J Clin Nutr 2009;89(4):1213-9.

20. Moreau M, Valente F, Mak R, Pelfrene E, de Smet P, De Backer G, et al. Obesity, body fat distribution and incidence of sick leave in the Belgian workforce: the Belstress study. Int J Obes Relat Metab Disord. 2004;28(4):574-82.

Correspondencia: Ricardo Antonio Agredo Zúñiga

Dirección: Universidad de San Buenaventura, Facultad de Ciencias de la Salud. Calle Real de Ternera 30-966. Cartagena, Colombia.

Teléfono: (552) 3177004726

Correo electrónico:fitsaludintegral@gmail.com 